\title{
A Seven-Dimensional System of the Navier-Stokes Equations for a Two-Dimensional Incompressible Fluid on a Torus
}

\author{
Heyuan Wang1, Yan Gao ${ }^{2}$ \\ ${ }^{1}$ College of Sciences, Liaoning University of technology, Jinzhou, China \\ ${ }^{2}$ Liaoning Petro-chemical Vocational Technology College, Jinzhou, China \\ Email: wangheyuan6400@sina.com, gaoyan67830@126.com
}

Received 29 August 2014; revised 29 September 2014; accepted 30 October 2014

Copyright @ 2014 by authors and Scientific Research Publishing Inc.

This work is licensed under the Creative Commons Attribution International License (CC BY).

http://creativecommons.org/licenses/by/4.0/

(c) (7) Open Access

\begin{abstract}
A seven-mode truncation system of the Navier-Stokes equations for a two-dimensional incompressible fluid on a torus is considered. Its stationary solutions and stability are presented; the existence of the attractor and the global stability of the system are discussed. The whole process, which shows a chaos behavior approached through instability of invariant tori, is simulated numerically by computers with the changing of Reynolds number. Based on numerical simulation results of bifurcation diagram, Lyapunov exponent spectrum, Poincare section, power spectrum and return map of the system, some basic dynamical behaviors of the new chaos system are revealed.
\end{abstract}

\section{Keywords}

The Navier-Stokes Equations, Strange Attractor, Lyapunov Function, Bifurcation, Chaos

\section{Introduction}

In recent years much attention has been devoted to the study of simple differential or difference equations, which although deterministic, exhibit a transition as some parameters go through certain values to a chaos behavior. The equations which are studied often arise in a natural way as simplified models in fluid dynamics and in ecology. The best known examples are perhaps the models of [1]-[3]. In these models a chaos behavior arises as a consequence of the appearance of an attractor of complicated structure which is called "strange attractor". Trajectories in a neighborhood of the attractor appear to move in a completely erratic way. Phenomena of this

How to cite this paper: Wang, H.Y. and Gao, Y. (2014) A Seven-Dimensional System of the Navier-Stokes Equations for a Two-Dimensional Incompressible Fluid on a Torus. Open Journal of Fluid Dynamics, 4, 347-362. 
kind are predicted under certain hypothesis, by the mathematical theory of turbulence of Ruelle and Takens [4]. In the following we consider a model obtained by a suitable seven-mode truncation of the Navier-Stokes equations for a 2-dimensional incompressible fluid on a torus [5]. The paper [5] has been published in Proceedings of the IWCFTA 2012, but the published paper is too rough and several deficiencies in the study of the published paper can be found. Only local results of dynamic behavior are given, and some simulation results are wrong; the transition to chaos is confused. This paper gives more accurate simulation results, discusses the dynamic behavior of the system at the high values of the Reynolds number, and analyses the evolution of the dynamic behavior of the system. This paper is extension and modification of the published article [5] and the content is more abundant. We argue on the basis of numerical simulation results analysis where in a certain range of the Reynolds number our system behaves in a way similar to that predicted by Ruelle and Takens. Dynamical behaviors of this new chaotic system, including some basic dynamical properties, bifurcations and routes to chaos, etc., have been investigated both theoretically and numerically by changing Reynolds number. Our purpose is to study how the phenomena of the model change when the number of modes in the truncation is slightly increased. The existence of the attractor and the global stability of the equations have been firmly verified and these theories can be used in other similar systems. Furthermore, some basic dynamical behaviors of the new chaos system, such as bifurcation diagram, Lyapunov exponent spectrum, Poincare section, power spectrum and return map, are presented.

\section{Seven-Mode Lorenz-Like Equations}

Consider the incompressible Navier-Stokes equations:

$$
\begin{gathered}
\frac{\partial u}{\partial t}+(u \cdot \nabla) u=-\nabla p+f+v \Delta u, \\
\nabla \cdot u=0, \\
\int_{T^{2}} u \mathrm{~d} X=0,
\end{gathered}
$$

on the torus $T^{2}=[0,2 \pi] \times[0,2 \pi]$, where $u$ is the velocity field, $p$ is the pressure and $f$ is a (periodic) volume force.

We expanded $u, f, p$ in Frourier series:

$$
\begin{aligned}
& u(X, t)=\sum_{K \neq 0} \mathrm{e}^{i K \cdot X} r_{K} \frac{K^{\perp}}{|K|} \\
& f(X, t)=\sum_{K \neq 0} \mathrm{e}^{i K \cdot X} f_{K} \frac{K^{\perp}}{|K|} \\
& p(X, t)=\sum_{K \neq 0} \mathrm{e}^{i K \cdot X} p_{K} \frac{K^{\perp}}{|K|}
\end{aligned}
$$

where $K=\left(k_{1}, k_{2}\right)$ is a "wave vector", with integer components, $K^{\perp}=\left(k_{2},-k_{1}\right), r_{K}=r_{K}(t)$ is a function of $t$, and the reality condition $r_{K}=-\bar{r}_{-K}$ holds. Substituting (4)-(6) into (1), we get formally the following equations for $\left\{r_{K}\right\}_{K \neq 0}$

$$
\dot{r}_{K}=-i \sum_{K_{1}+K_{2}+K=0} \frac{K_{1}^{\perp} \cdot K_{2}\left(K_{2}^{2}-K_{1}^{2}\right)}{2|K|\left|K_{1}\right|\left|K_{2}\right|} \bar{r}_{K_{1}} \bar{r}_{K_{2}}-v|K|^{2} r_{K}+f_{K}
$$

where $L$ is a set of wave vectors such that if $K \in L$, also $-K \in L$.

We take as the set of vectors $K_{1}=(1,-1), \quad K_{2}=(0,3), \quad K_{3}=(1,2), \quad K_{4}=(1,-2), \quad K_{5}=(0,1)$, $K_{6}=(1,0), K_{7}=(2,1)$, and their opposites, namely $L=\left\{ \pm K_{1}, \pm K_{2}, \pm K_{3}, \pm K_{4}, \pm K_{5}, \pm K_{6}, \pm K_{7}\right\}$. Let $v=1$, and make the following transform 


$$
\left\{\begin{array}{l}
r_{K_{1}}=2 \sqrt{10} x_{1}, r_{K_{2}}=2 \sqrt{10} i x_{2}, r_{K_{3}}=2 \sqrt{10} x_{3}, r_{K_{4}}=2 \sqrt{10} i x_{4}, \\
r_{K_{5}}=2 \sqrt{10} x_{5}, r_{K_{6}}=2 \sqrt{10} i x_{6}, r_{K_{7}}=-2 \sqrt{10} i x_{7} .
\end{array}\right.
$$

Taking the force acting on the mode $K_{3}$, and let $r=\sum_{k=K_{1}}^{K_{7}}\left|f_{K}\right|=\left|f_{K_{3}}\right|=R e$ (Reynolds number), with a lot of Calculation we obtain the following system

$$
\left\{\begin{array}{l}
\dot{x}_{1}=-2 x_{1}+4 x_{2} x_{3}+4 x_{4} x_{5} \\
\dot{x}_{2}=-9 x_{2}+3 x_{1} x_{3} \\
\dot{x}_{3}=-5 x_{3}-7 x_{1} x_{2}+\frac{9 \sqrt{5}}{5} x_{1} x_{7}+r \\
\dot{x}_{4}=-5 x_{4}-x_{1} x_{5} \\
\dot{x}_{5}=-x_{5}-3 x_{1} x_{4}+\sqrt{5} x_{1} x_{6} \\
\dot{x}_{6}=-x_{6}-\sqrt{5} x_{1} x_{5} \\
\dot{x}_{7}=-5 x_{7}-\frac{9 \sqrt{5}}{5} x_{1} x_{3}
\end{array}\right.
$$

\section{The Stationary Solution and Their Stability Properties}

In this section we discuss the stationary solution and their stability properties of the system (2.8). Let

$$
F(X, r)=\left(\begin{array}{c}
-2 x_{1}+4 x_{2} x_{3}+4 x_{4} x_{5} \\
-9 x_{2}+3 x_{1} x_{3} \\
-5 x_{3}-7 x_{1} x_{2}+\frac{9 \sqrt{5}}{5} x_{1} x_{7}+r \\
-5 x_{4}-x_{1} x_{5} \\
-x_{5}-3 x_{1} x_{4}+\sqrt{5} x_{1} x_{6} \\
-x_{6}-\sqrt{5} x_{1} x_{5} \\
-5 x_{7}-\frac{9 \sqrt{5}}{5} x_{1} x_{3}
\end{array}\right),
$$

setting $F(X, r)=0$ we can find out stationary solutions of the system (8). In the following we present stability properties

(a) For $0 \leq r<R_{1}=\frac{5 \sqrt{6}}{2}$ there is only one stationary solution $P_{0}=\left(0,0, \frac{r}{5}, 0,0,0,0\right)$, which turns out to be stable for $r$ small enough (this is a particular case of general results on the theory of the Navier-Stokes equations [6]), and numerical evidence suggests that the above solution is a global attractor.

(b) For $R_{1}<r<67.54$ there are 3 stationary solutions: the old one $P_{0}$, which has become unstable (as a consequence of the crossing of the imaginary axis by one of the eigenvalues of the Lyapunov matrix) and two additional $P_{ \pm}$as follow

$$
\left(\sigma 5 \sqrt{\frac{\sqrt{6}(2 r-5 \sqrt{6})}{836}}, \sigma \frac{5 \sqrt{6}}{6} \sqrt{\frac{\sqrt{6}(2 r-5 \sqrt{6})}{836}}, \frac{\sqrt{6}}{2}, 0,0,0,-\sigma \frac{9 \sqrt{30}}{10} \sqrt{\frac{\sqrt{6}(2 r-5 \sqrt{6})}{836}}\right)
$$

where $\sigma= \pm 1$, and they are stable. Numerical evidences indicate that any randomly chosen initial data is attracted by them, so they are global attractors. When $r \geq 114.685$, a pair of complex conjugate eigenvalues crosses the imaginary axis, so we have the following conclusion.

(c) For $r>114.685$, all the stationary solutions of (8) become unstable. 


\section{The Existence of Attractor and Analysis of Global Stability}

In the following we prove the existence of attractor of the system (8).

By calculating $(8.1) \times x_{1}+(8.2) \times x_{2}+(8.3) \times x_{3}+(8.4) \times x_{4}+(8.5) \times x_{5}+(8.6) \times x_{6}+(8.7) \times x_{7}$ we get

$$
\begin{aligned}
& \dot{x}_{1} x_{1}+2 x_{1}^{2}+\dot{x}_{2} x_{2}+9 x_{2}^{2}+\dot{x}_{3} x_{3}+5 x_{3}^{2}+\dot{x}_{4} x_{4}+5 x_{4}^{2} \\
& +\dot{x}_{5} x_{5}+x_{5}^{2}+\dot{x}_{6} x_{6}+x_{6}^{2}+\dot{x}_{7} x_{7}+5 x_{7}^{2}=x_{3} r
\end{aligned}
$$

accordingly,

$$
\frac{1}{2} \frac{\mathrm{d}}{\mathrm{d} t}\left(x_{1}^{2}+x_{2}^{2}+x_{3}^{2}+x_{4}^{2}+x_{5}^{2}+x_{6}^{2}+x_{7}^{2}\right)+\left(2 x_{1}^{2}+9 x_{2}^{2}+5 x_{3}^{2}+5 x_{4}^{2}+x_{5}^{2}+x_{6}^{2}+5 x_{7}^{2}\right)=x_{3} r .
$$

letting $|u(t)|^{2}=x_{1}^{2}+x_{2}^{2}+x_{3}^{2}+x_{4}^{2}+x_{5}^{2}+x_{6}^{2}+x_{7}^{2}$, and using Young Inequality we get

$$
\frac{1}{2} \frac{\mathrm{d}}{\mathrm{d} t}\left(x_{1}^{2}+x_{2}^{2}+x_{3}^{2}+x_{4}^{2}+x_{5}^{2}+x_{6}^{2}+x_{7}^{2}\right)+\left(2 x_{1}^{2}+9 x_{2}^{2}+5 x_{3}^{2}+5 x_{4}^{2}+x_{5}^{2}+x_{6}^{2}+5 x_{7}^{2}\right)=x_{3} r \leq \frac{r^{2}}{4}+x_{3}^{2},
$$

as a result, $\frac{\mathrm{d}}{\mathrm{d} t}|u|^{2}+2|u|^{2} \leq \frac{r^{2}}{2}$. Using the Gronwall Inequality [7] we get

$$
|u|^{2} \leq|u(0)|^{2} \mathrm{e}^{-2 t}+\frac{r^{2}}{4}\left(1-\mathrm{e}^{-4 t}\right)
$$

, then

$$
\limsup _{t \rightarrow \infty}|u(t)|^{2} \leq \frac{r^{2}}{4}
$$

From above we have

$$
\limsup _{t \rightarrow \infty}|u(t)| \leq \frac{r}{2}=\rho_{0} .
$$

If $\rho$ big enough, $B(0, \rho)$ is an not only functional invariant set but also absorbing set. As a result the system (8) has the global attractor [7] [8].

When the system is a global stability,its orbits contract into a domain called the trapping region. Therefore, if the existence of the trapping region is proved, the system has the global stability, though the stationary solutions are unstable. We construct a following Liapunov function of the system (8)

$$
V\left(x_{1}, x_{2}, x_{3}, x_{4}, x_{5}, x_{6}, x_{7}\right)=x_{1}^{2}+x_{2}^{2}+x_{3}^{2}+x_{4}^{2}+x_{5}^{2}+x_{6}^{2}+x_{7}^{2}>0
$$

Setting $V\left(x_{1}, x_{2}, x_{3}, x_{4}, x_{5}, x_{6}, x_{7}\right)=k$, obviously, when $k$ is a positive constant, the Equation (9) represents a sphere, which is labeled as $E$. By calculating we obtain the following derivative

$$
\begin{aligned}
\frac{\mathrm{d} V}{\mathrm{~d} t} & =2 x_{1} \dot{x}_{1}+2 x_{2} \dot{x}_{2}+2 x_{3} \dot{x}_{3}+2 x_{4} \dot{x}_{4}+2 x_{5} \dot{x}_{5}+2 x_{6} \dot{x}_{6}+2 x_{7} \dot{x}_{7} \\
& =-2\left(2 x_{1}^{2}+9 x_{2}^{2}+5 x_{3}^{2}+5 x_{4}^{2}+x_{5}^{2}+x_{6}^{2}+x_{7}^{2}-x_{3} r\right) \\
& =-2\left[2 x_{1}^{2}+9 x_{2}^{2}+5\left(x_{3}-\frac{r}{10}\right)^{2}+5 x_{4}^{2}+x_{5}^{2}+x_{6}^{2}+x_{7}^{2}-\frac{r^{2}}{20}\right]
\end{aligned}
$$

Obviously $2 x_{1}^{2}+9 x_{2}^{2}+5\left(x_{3}-\frac{r}{10}\right)^{2}+5 x_{4}^{2}+x_{5}^{2}+x_{6}^{2}+x_{7}^{2}-\frac{r^{2}}{20}=0$ represents an ellipsoid in $R^{7}$, which is labeled as $C$. From (10) We know that $\frac{\mathrm{d} V}{\mathrm{~d} t}<0$ on outside of $C, \frac{\mathrm{d} V}{\mathrm{~d} t}=0$ on $C$, and $\frac{\mathrm{d} V}{\mathrm{~d} t}>0$ inside of $C$. If $k$ 
is big enough, $E$ will include $C$. Therefore, from (10) we know that $\frac{\mathrm{d} V}{\mathrm{~d} t}<0, V \frac{\mathrm{d} V}{\mathrm{~d} t}<0$ on outside of $C$.

From the Liapunov theory we know that the orbits out of system (8) will enter E. Namely E is the trapping region of the Equations (8). Though the stationary solutions $P_{0}, P_{ \pm}$are all unstable, the system (8) still has the global stability. orbits of system contract into the trapping region and oscillates in the trapping region. Finally the orbits form an invariant set in the trapping region, which is called the attractor.

\section{Numerical Simulation}

With the increasing of Reynolds number $r$, the stability of the Equation (8) will change, and some nonlinear phenomena appear, such as the Hopf bifurcation and the chaos. In this section, we present the numerical simulation results of dynamical behavior of the system (8).

1) At $r<114.685$ the stationary solutions $P_{ \pm}$of (8) is stable, Numerical evidences indicate that any randomly chosen initial data is attracted by one of them, so they are global attractors(Figures 1-4).

2) When $r=114.685$, the stationary solutions $P_{ \pm}$of (8) become unstable because a pair of complex conjugate eigenvalues of Jacobian matrix at stationary point $P_{ \pm}$cross the imaginary axis, and the stable periodic orbits $\zeta_{+}$and $\zeta_{-}$around the fixed points $P_{ \pm}$arise via a Hopf bifurcation, and they are stable up to $r=143.463$ and numerical results shows that they attract any point chosen at random (Figure 5 and Figure 6).

3) At $r=143.463$, the periodic orbits lose stability. As predicted by the general theory of bifurcation [3], the Numerical Simulation shows that two attracting tori $T\left(\zeta_{+}\right)$and $T\left(\zeta_{-}\right)$arise from the two periodic orbits $\zeta_{+}$ and $\zeta_{-}$(Figure 7 and Figure 8 ).

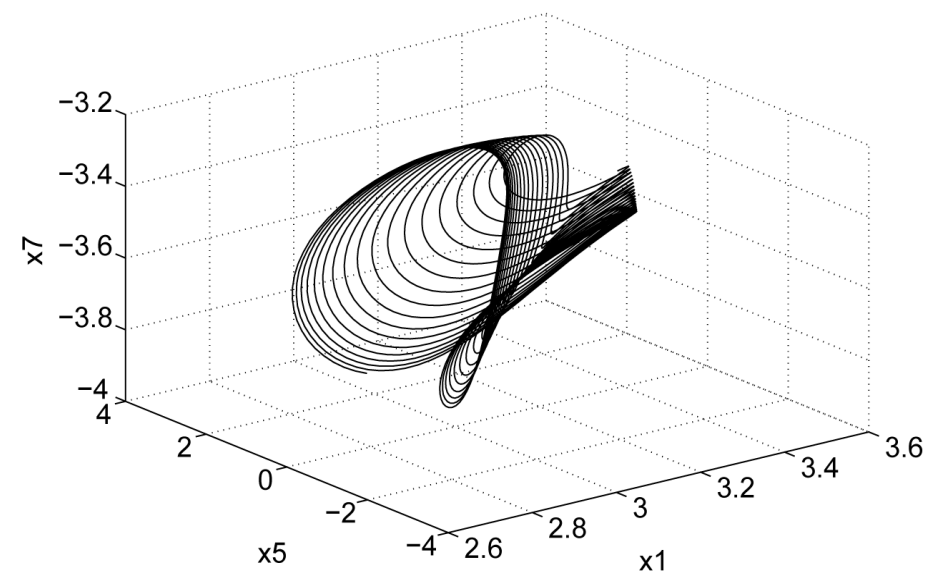

Figure 1. $\left(P_{ \pm}\right.$is stable for $\left.r<114.685\right)$.

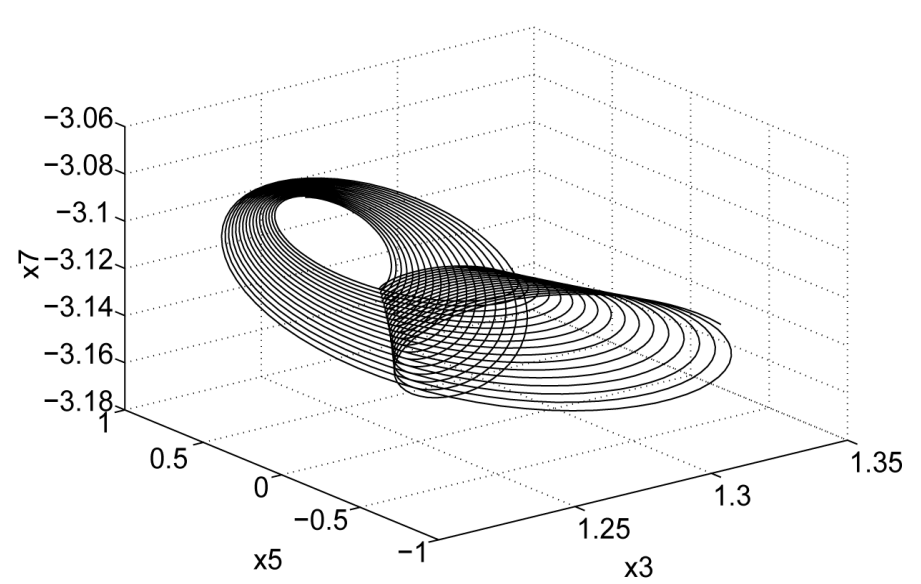

Figure 2. $\left(P_{ \pm}\right.$is stable for $\left.r<114.685\right)$. 


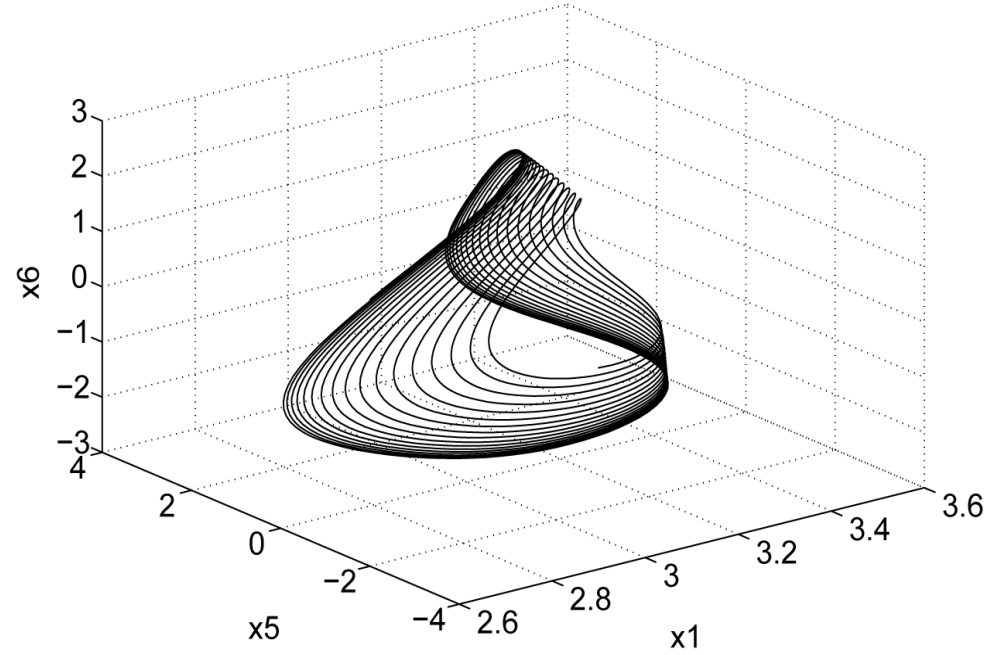

Figure 3. $\left(P_{ \pm}\right.$is stable for $\left.r<114.685\right)$.

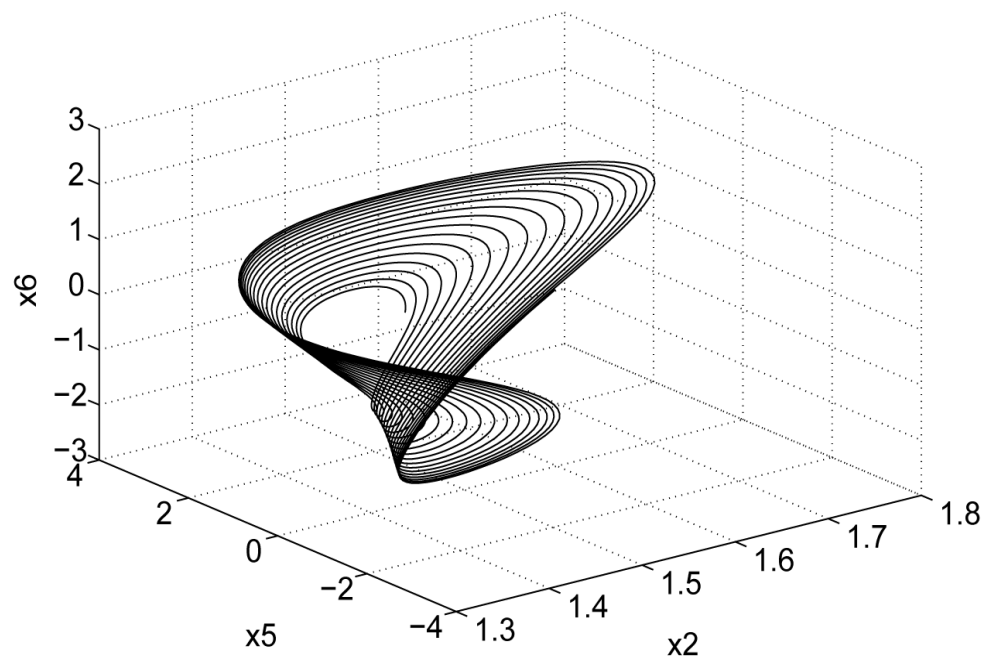

Figure 4. $\left(P_{ \pm}\right.$is stable for $\left.r<114.685\right)$.

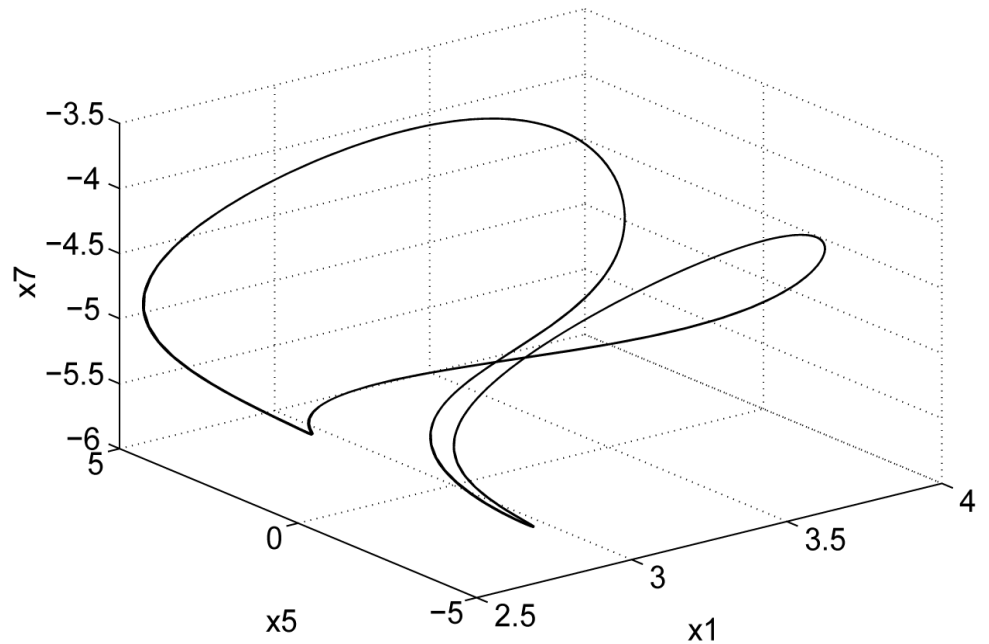

Figure 5. (Periodic orbit for $r=114.685$ ). 


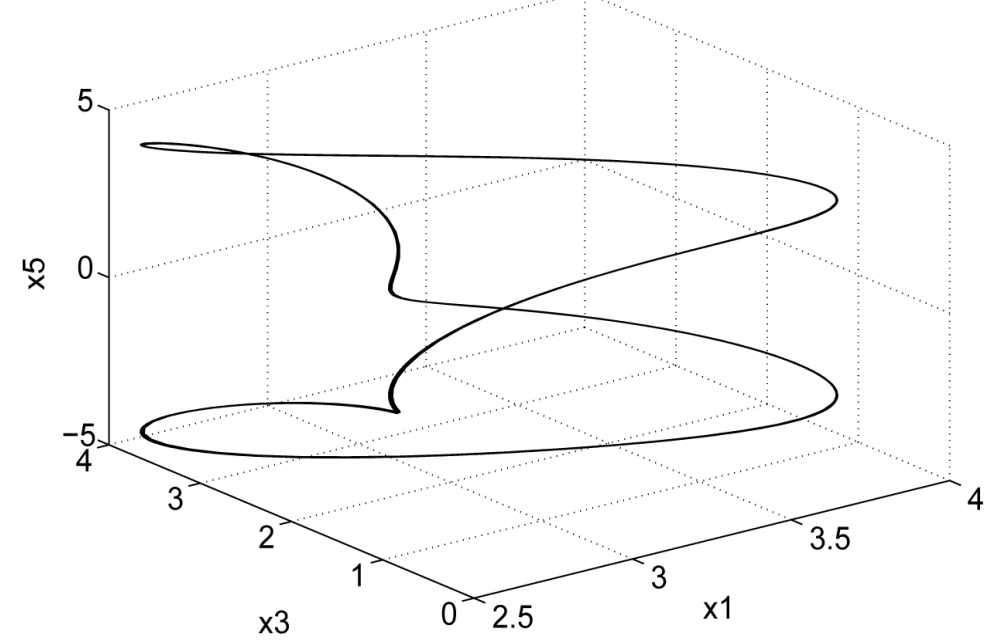

Figure 6. (Periodic orbit for $r=114.685$ ).

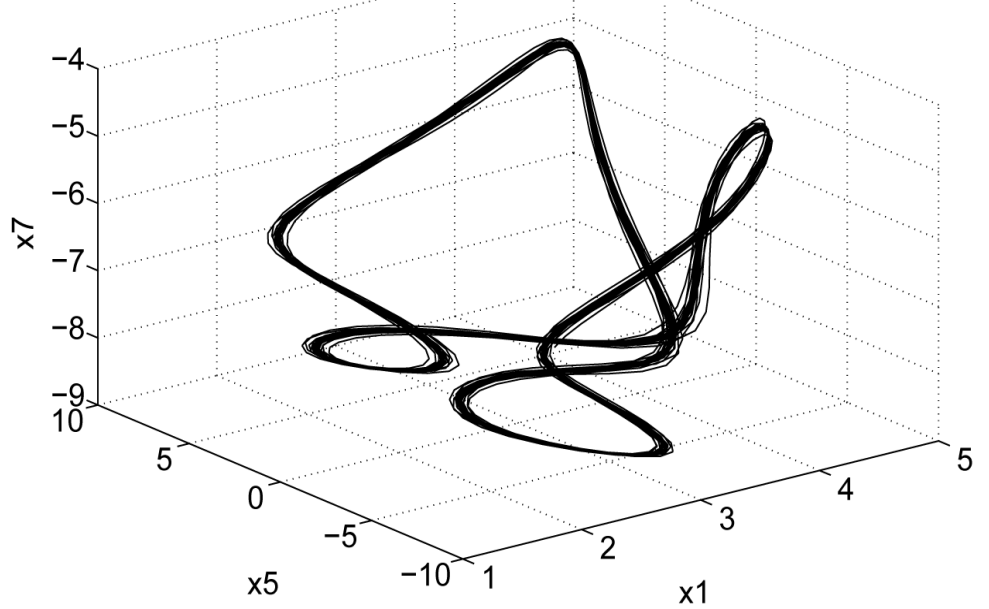

Figure 7. (The attracting tori for $r=143.463$ ).

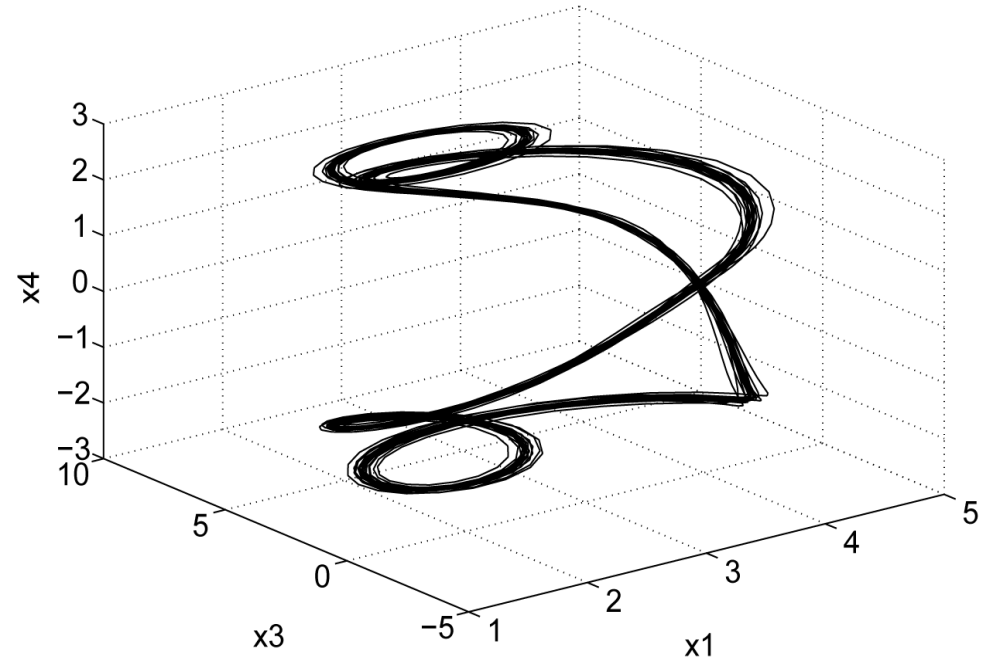

Figure 8. (The attracting tori for $r=143.463$ ). 
4) With the increasing of the Reynolds number $r$, a strong hysteresis phenomenon(i.e., coexistence of stable attractors) appears, in some intervals hysteresis takes place between closed orbits and tori (Figures 9-19).

5) At $r=158.631$, these attracting toris lose stability, the strange attractor appears (Figures 20-25).

6) Figure 26 and Figure 27 show Bifurcation diagrams and the largest Lyapunov exponents of the system (2.8).

7) Figures 28-30 show Poincare section, return map and power spectrum of the system (2.8) when $r=184$, they indicate chaos behavior feature of the new chaos system.

8) For $r>158.631$ all stable orbits disappears, by studying the flow of a randomly chosen initial point, trajectories are observed which all appear completely chaotic and sensitively dependent on initial conditions. The entire process repeats itself indefinitely. This situation appears analogous to that found by Curry in [4], where the flow in the turbulent parameter range is driven by a similar mechanism, When $r$ increases, the behavior of our system becomes more complicated (Figures 20-25).

\section{Conclusions}

In this work we have reported the results of our theoretical and numerical investigation on a model of seven nonlinear ordinary differential equations. Such a model, obtained by a suitable seven-mode truncation of the Navier-Stokes equations for an incompressible fluid on a torus, exhibits a very varied phenomenology, with an

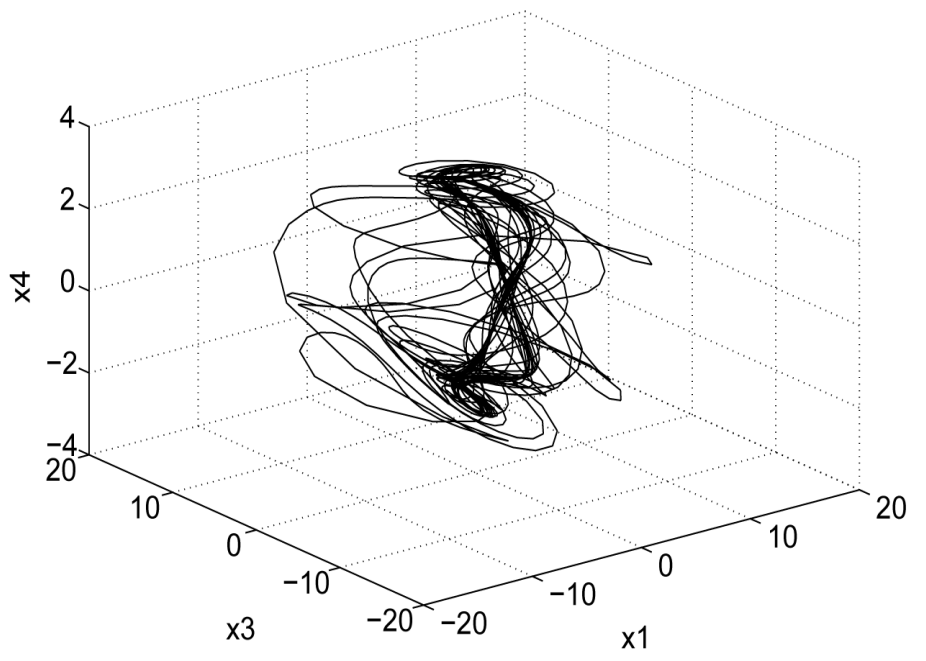

Figure 9. (Quasi-periodic orbit for $r=148.685$ ).

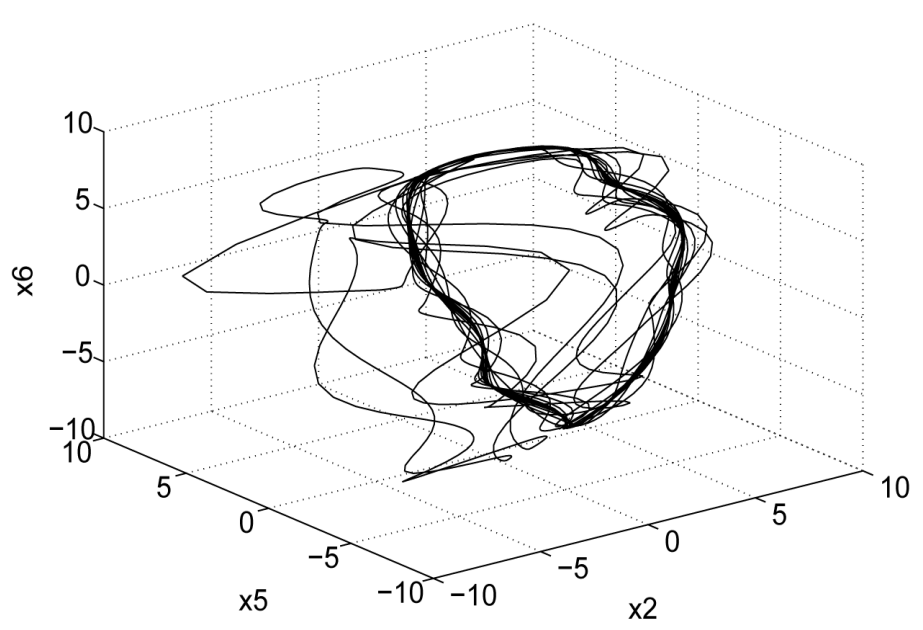

Figure 10. (Quasi-periodic orbit for $r=148.685)$. 


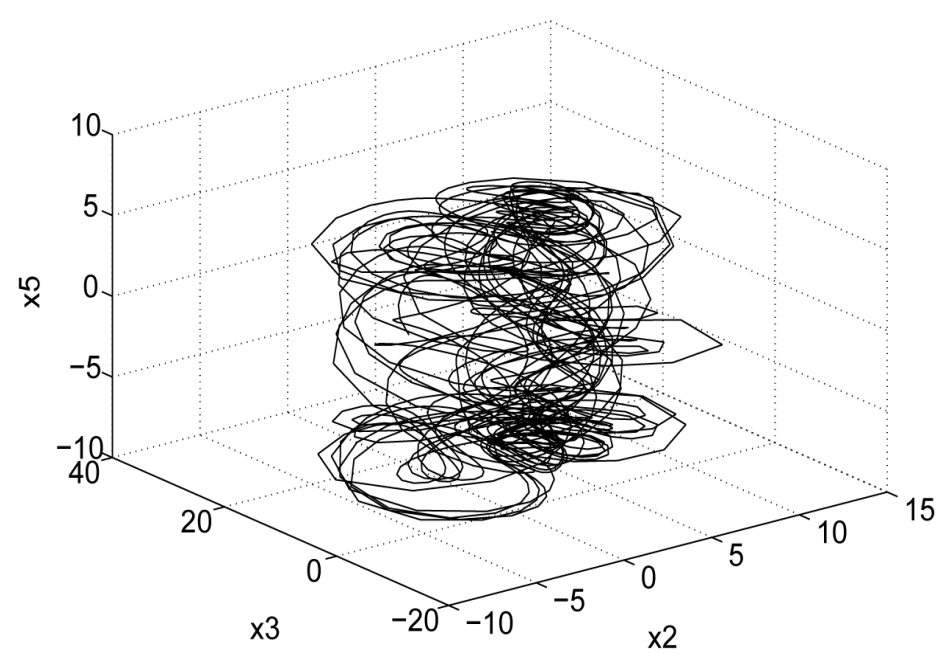

Figure 11. (Quasi-periodic orbit for $r=149.35$ ).

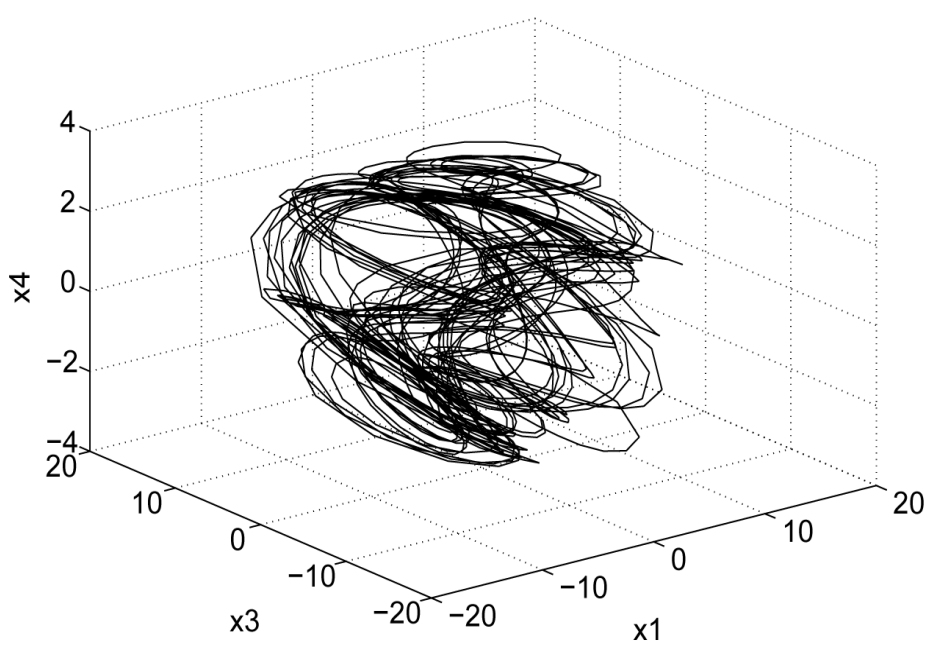

Figure 12. (Quasi-periodic orbit for $r=151.35$ ).

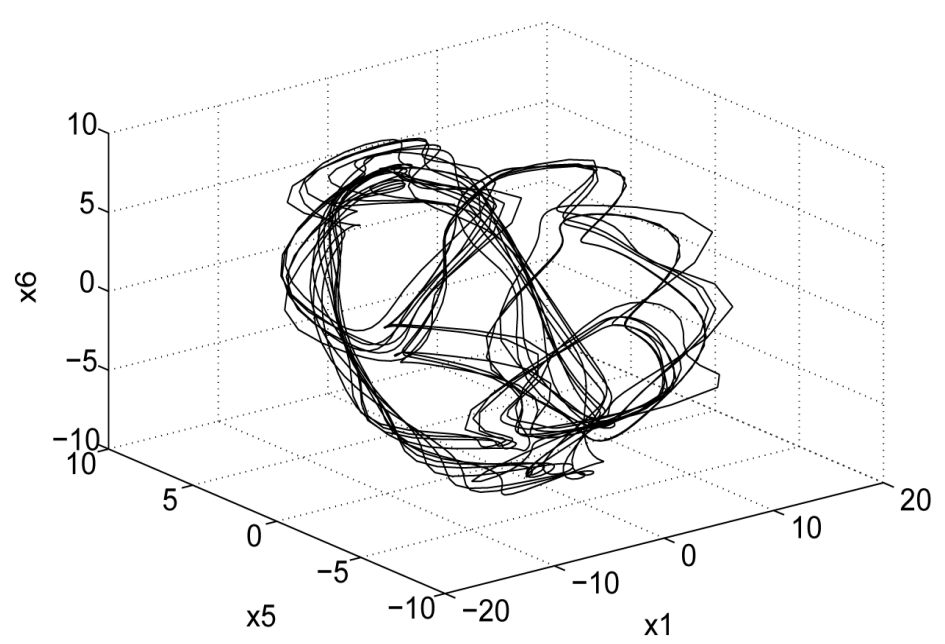

Figure 13. (Quasi-periodic orbit for $r=152.65$ ). 


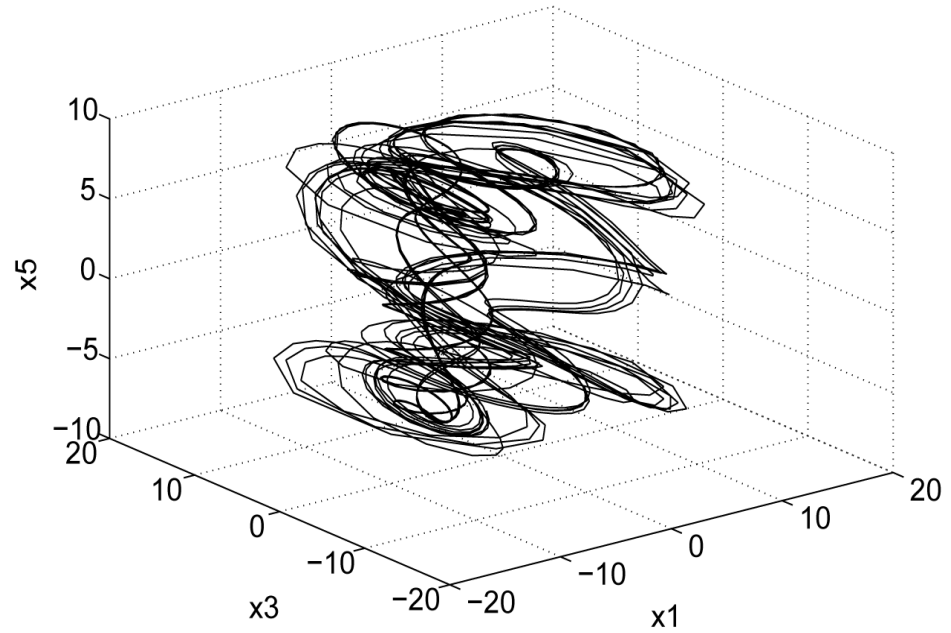

Figure 14. (Quasi-periodic orbit for $r=154.35$ ).

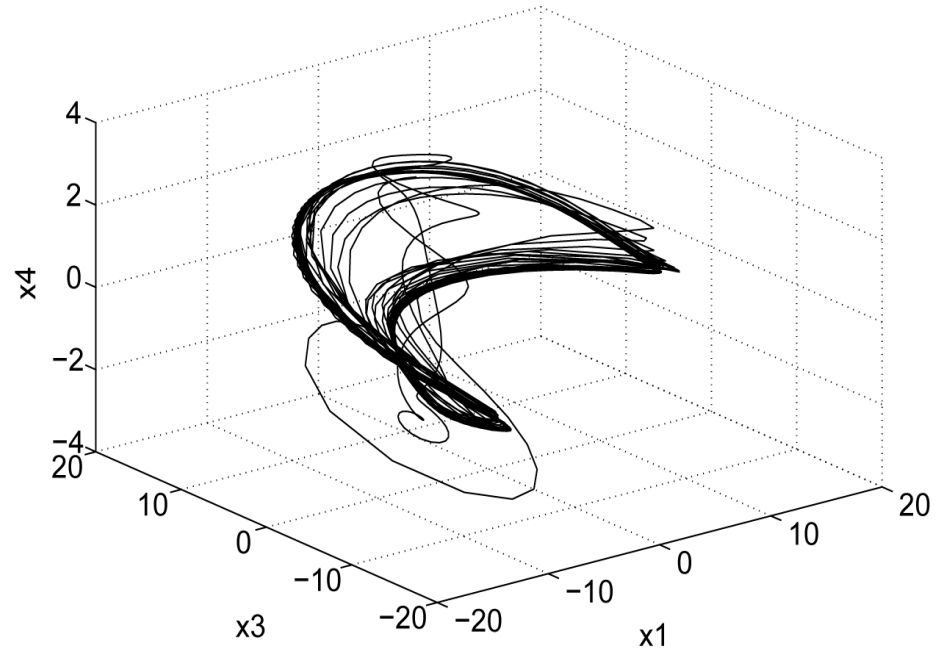

Figure 15. (Quasi-periodic orbit for $r=155.12$ ).

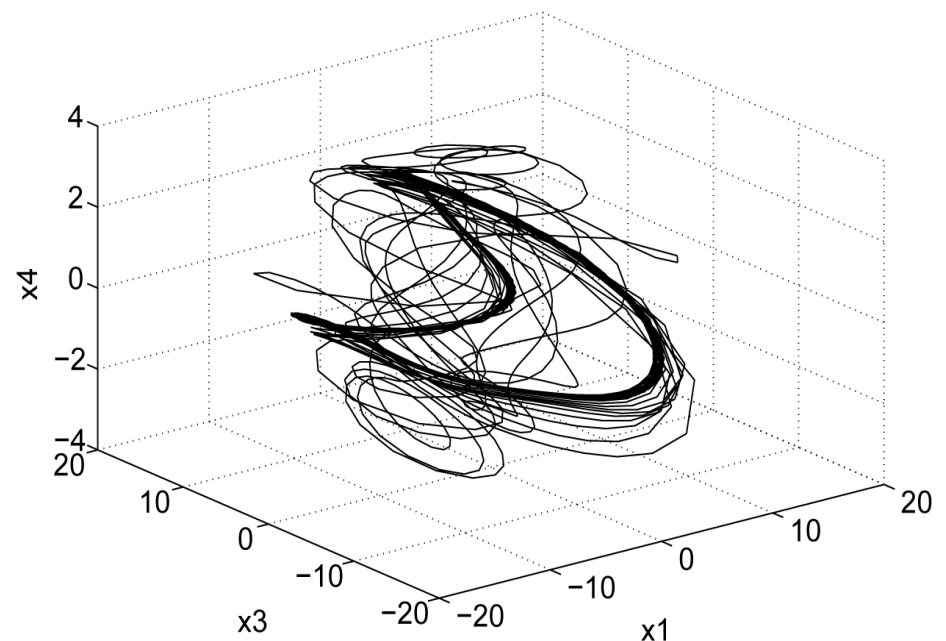

Figure 16. (Quasi-periodic orbit for $r=156.43$ ). 


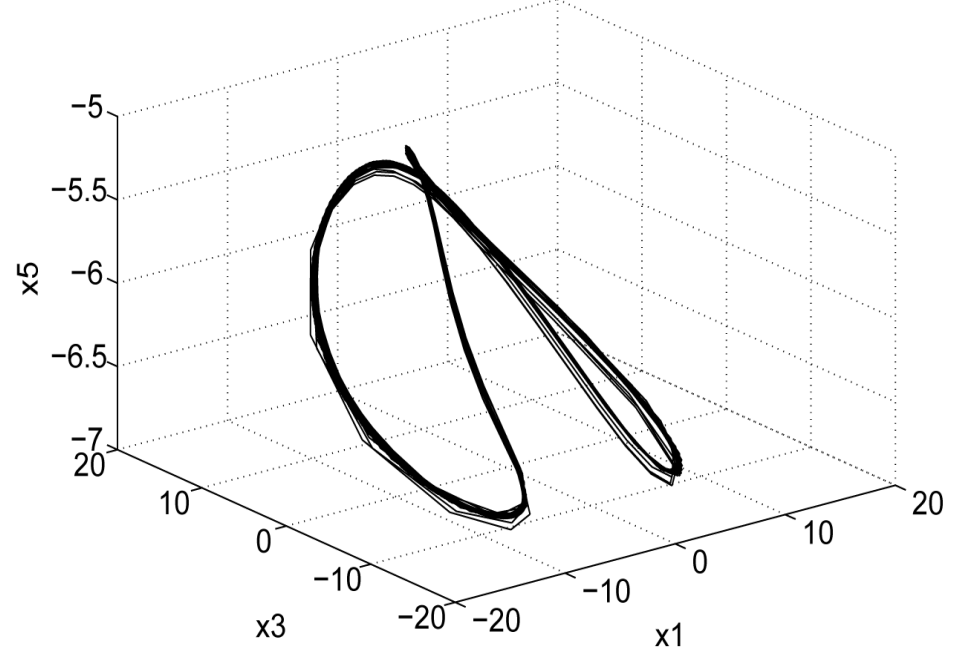

Figure 17. (The attracting tori for $r=156.43$ ).

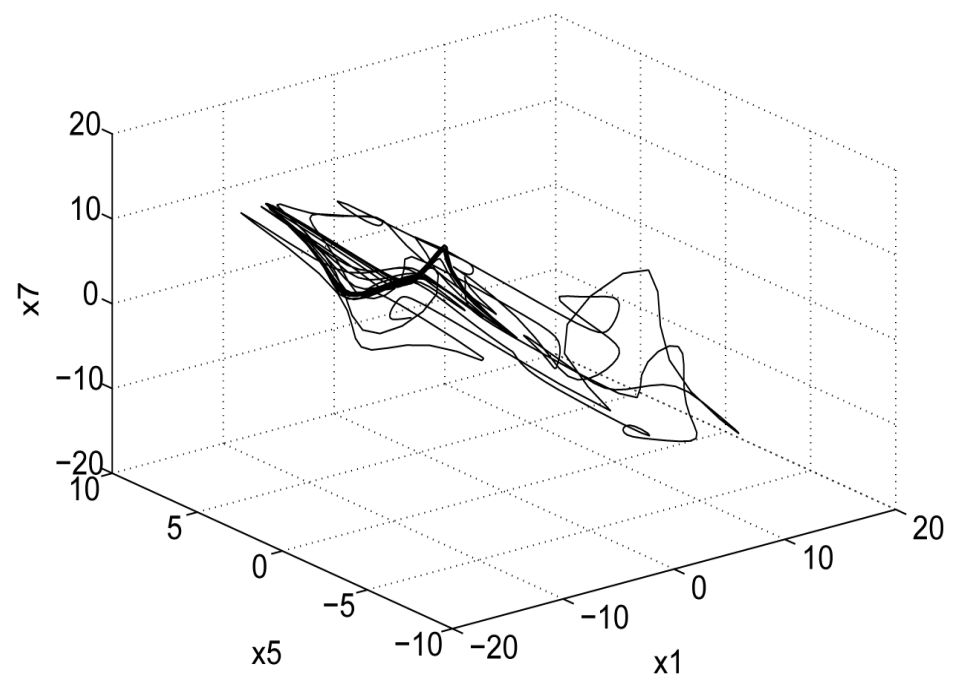

Figure 18. (Quasi-periodic orbit for $r=156.83$ ).

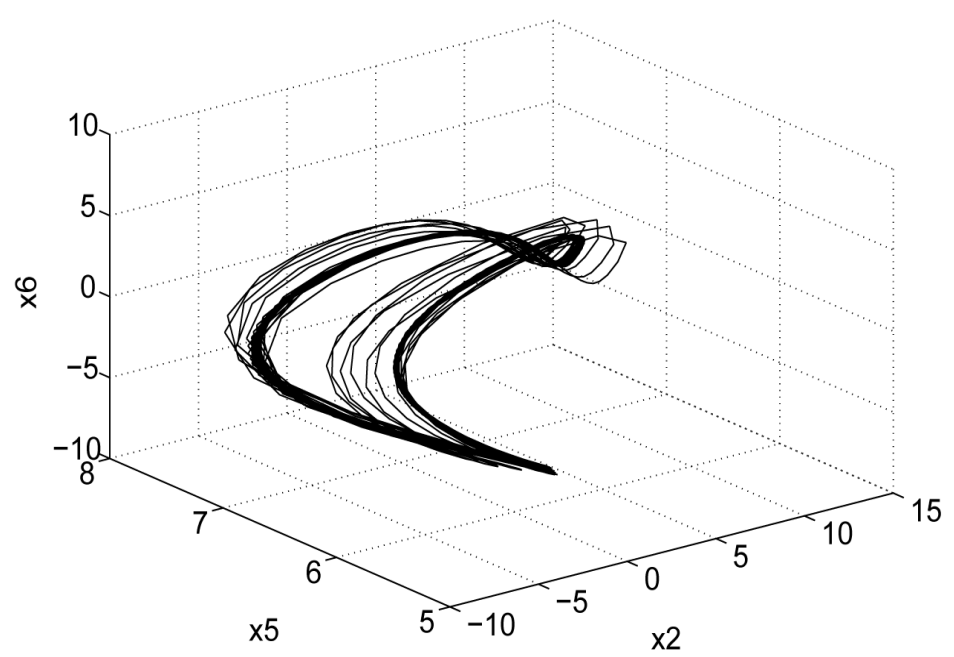

Figure 19. (The attracting tori for $r=157.23$ ). 


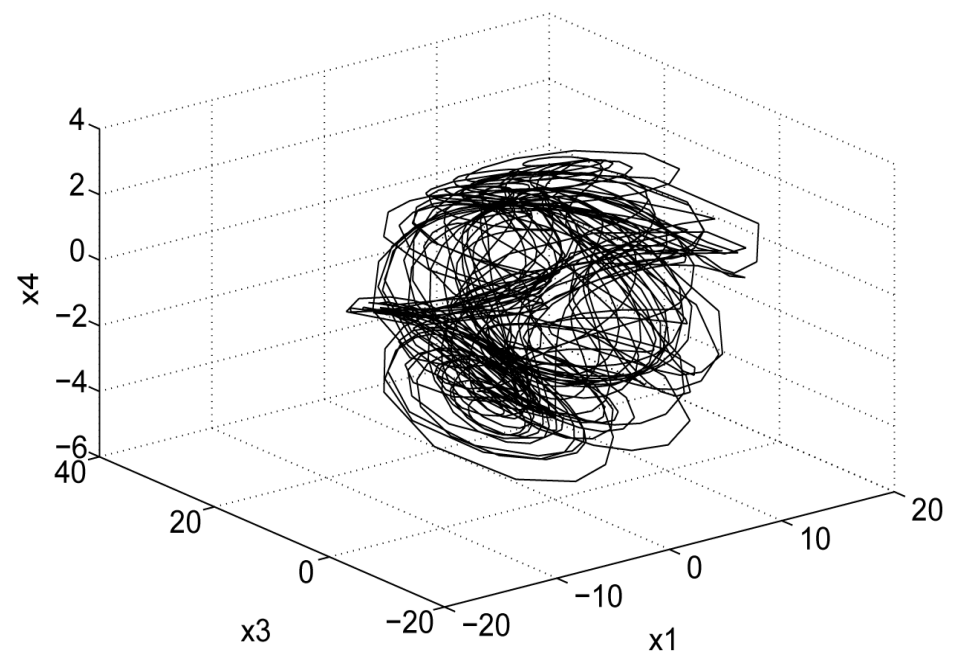

Figure 20. (The strange attractor for $r=158.5$ ).

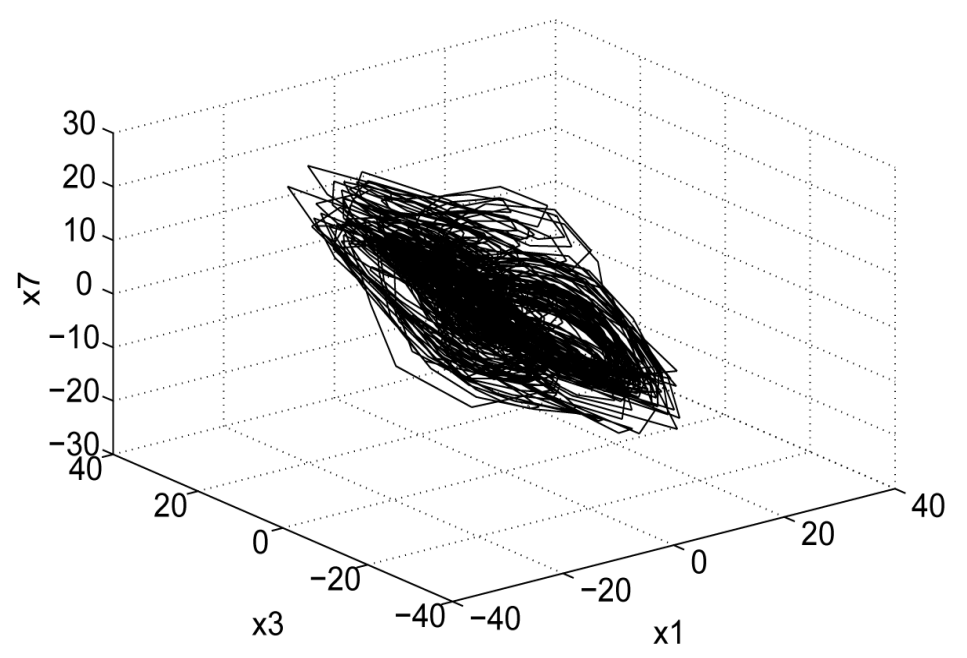

Figure 21. (The strange attractor for $r=159.5$ ).

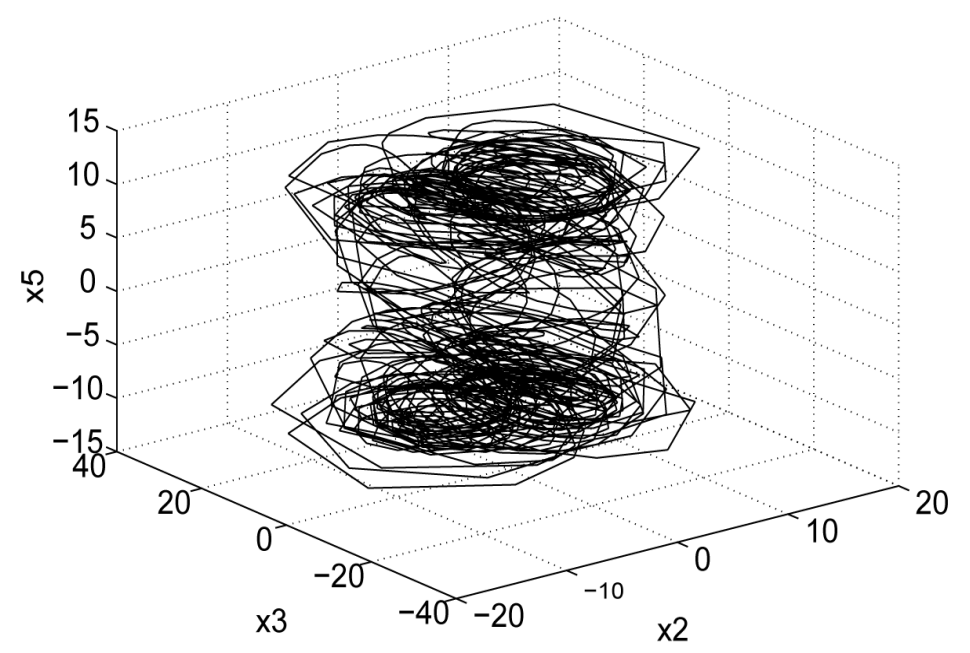

Figure 22. (The strange attractor for $r=159.5$ ). 


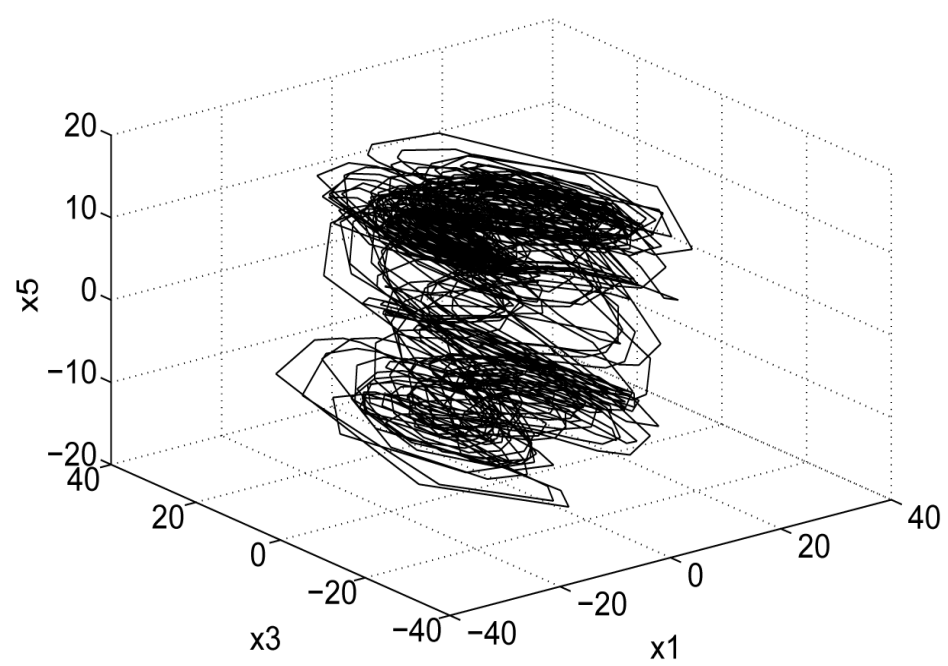

Figure 23. (The strange attractor for $r=160.5$ ).

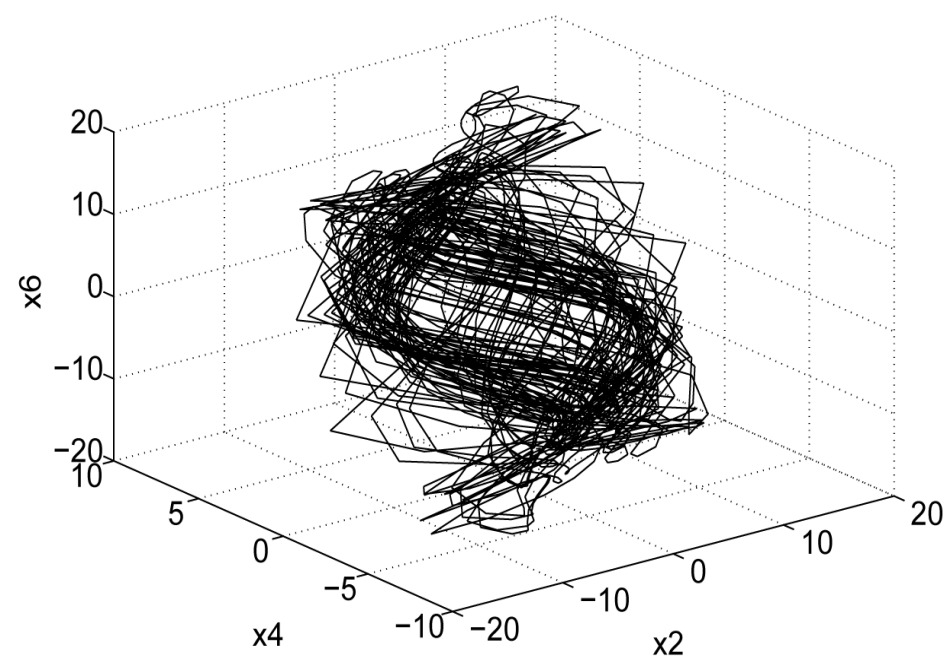

Figure 24. (The strange attractor for $r=250.5$ ).

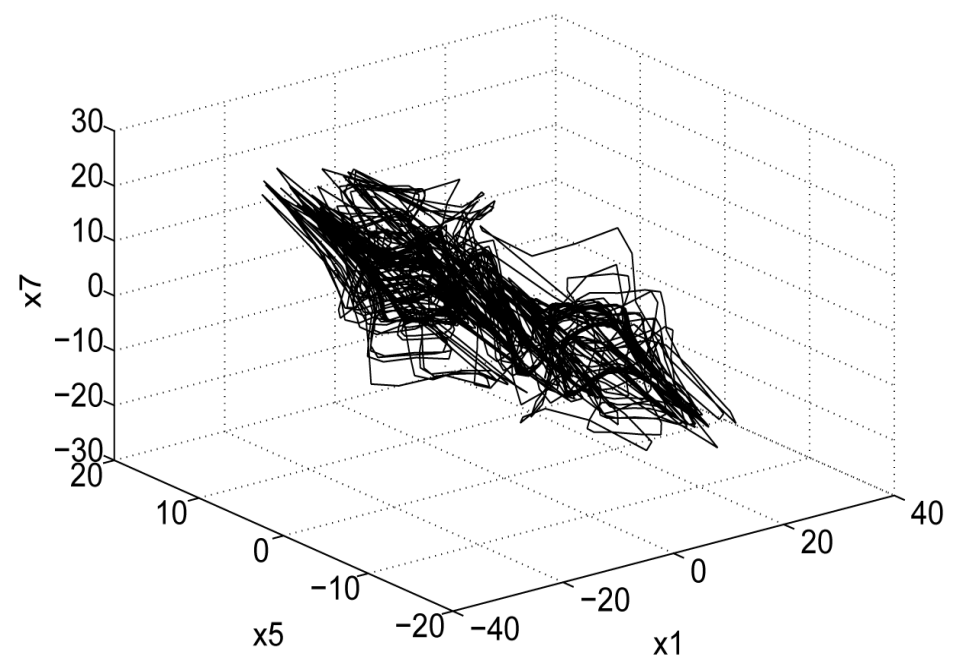

Figure 25. (The strange attractor for $r=360.5$ ). 


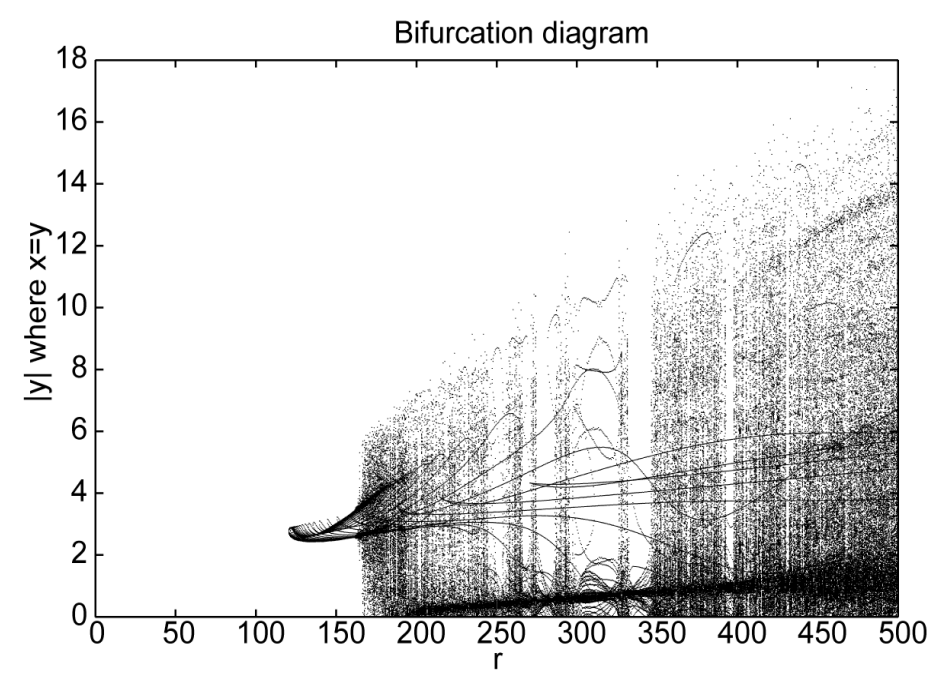

Figure 26. (Bifurcation diagrams).

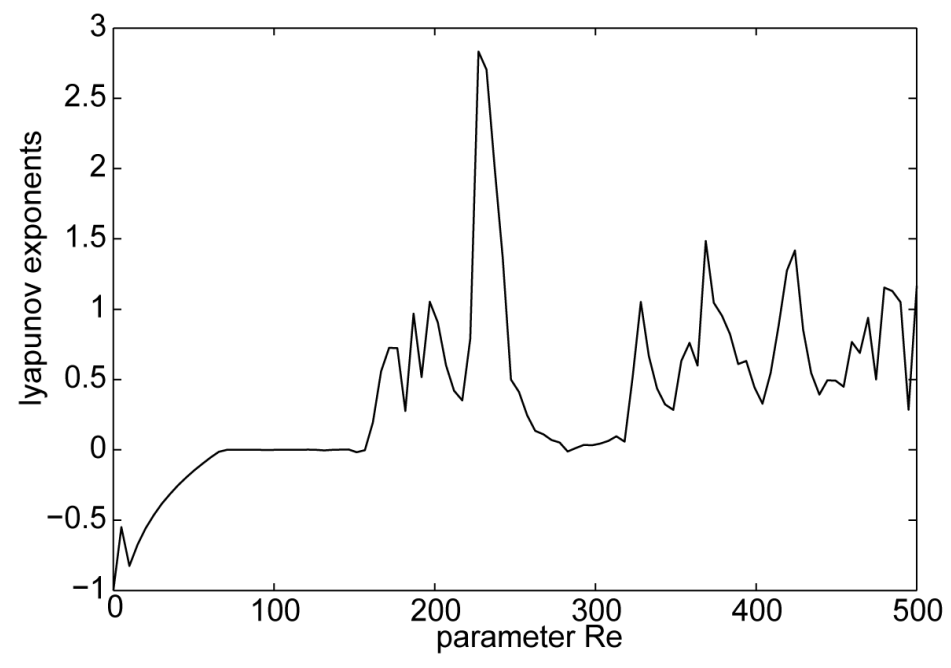

Figure 27. (The largest Lyapunov exponents).

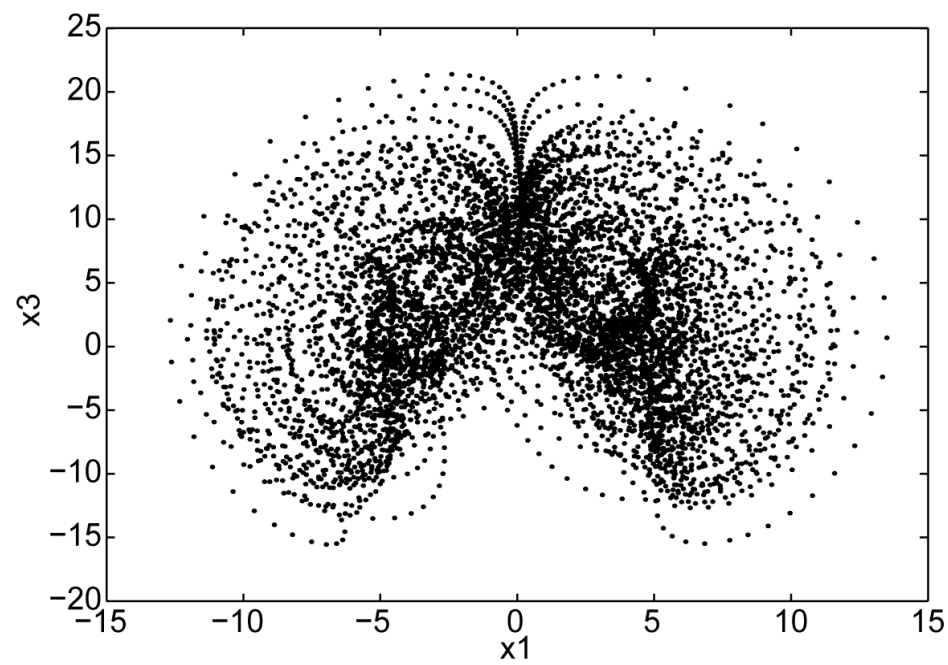

Figure 28. (The Poincare section for $r=184$ ). 


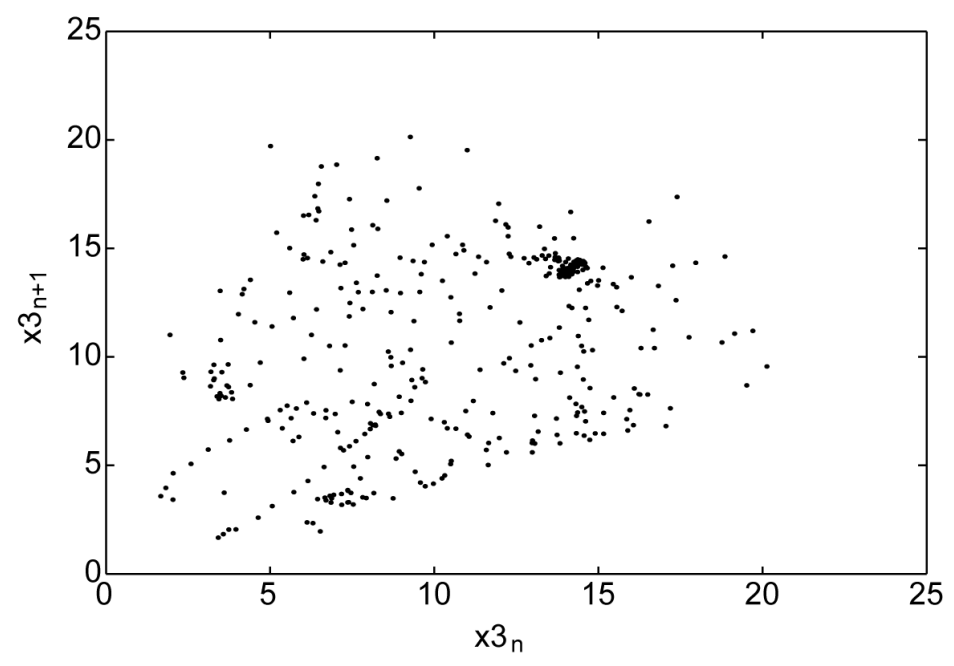

Figure 29. (The return map for $r=184$ ).

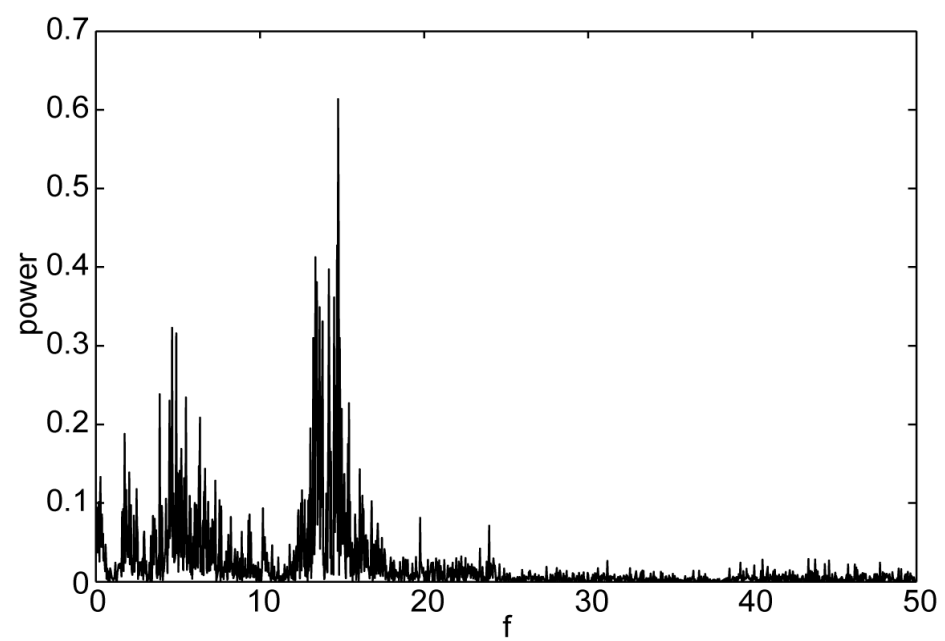

Figure 30. (The power spectrum for $r=184$ ).

interesting sequence of bifurcations. From numerical results we present four different and independent stories describing the complete phenomenology of the model. The first story consists of a sequence of a bifurcations very similar to the one found by Curry in [4]: The fixed point $P_{0}$ bifurcates to the two fixed $P_{ \pm}$; via a direct Holf bifurcation $P_{+}$and $P_{-}$bifurcate to the periodic orbits $\zeta_{+}$and $\zeta_{-}$, which on their turn bifurcate to the tori $T\left(\zeta_{+}\right)$and $T\left(\zeta_{-}\right)$. The further three stories show three interesting examples of "life" of orbit (Figure 9, Figure 14 and Figure 18), each one with its own characteristics. A remark feature concerns a strong phenomenon of hysteresis (i.e, coexistence of stable attractors) characterizing the model (Figures 9-19); three different stable orbits are present in some intervals, and hysteresis takes place between closed orbits and tori in the intervals.

For all the values of the Reynolds number $r$ larger than 158.631, when no stable periodic orbits or tori are present any more, the model exhibits a turbulent behavior. In fact any randomly chosen point describes trajectories which appear to be completely random and sensitively dependent on initial conditions. Since all the numerical investigations carried up to $r=5000$ keep showing a stochastic behavior, we think that turbulence might also persist for $r$ tending to infinity and that no stable attracting periodic orbit exists at the high values of the Reynolds number. Our seven-mode system does not reproduce the qualitative features of the five-mode model from which it has been obtained as an extension. This result makes more striking what appears already in [4], where a 14-mode generalization of the three-mode Lorenz system is presented: new modes can change quite completely the phenomenology of a model. 


\section{Acknowledgements}

We thank the editor and the referee for their comments. Research work is funded by the funds for education department of Liaoning Province (L2013248) and science and technology funds of Jinzhou city (13A1D32).

\section{References}

[1] Wang, H.Y. (2012) Dynamical Behaviors and Numerical Simulation of Lorenz Systems for the Incompressible Flow between Two Concentric Rotating Cylinders. International Journal of Bifurscation and Chaos, 22, 1-11.

[2] Wang, H.Y. and Jiang, H.B. (2007) Bifurcation Analysis of the Model System Similar to the Lorenz Equations of the Flow between Two Concentric Rotating Spheres. Numerical Mathematics: A Journal of Chinese Universities English Series, 29, 278-288.

[3] Franceschini, V. and Zanasi, R. (1992) Three-Dimensional Navier-Stokes Equations Truncated on a Torus. Nonlinearity, 4, 189-209. http://dx.doi.org/10.1088/0951-7715/5/1/008

[4] Curry, J.H. (1978) A Generalized Lorenz System. Communications in Mathematical Physics, 60, 193-204. http://dx.doi.org/10.1007/BF01612888

[5] Wang, H.Y. and Gao, Y. (2012) The Dynamical Behaviors and Numerical Simulation of a Seven-Mode Truncation System of the Navier-Stokes Equations for a Two-Dimensional Incompressible Fluid on a Torus. Proceedings of the IWCFTA 2012, 54-57.

[6] Ladyzhenskaya, O.A. (1969) The Mathematical Theory of Viscous Incompressible Flows. Gordon and Breach, New York.

[7] Li, K.T. and Ma, Y.C. (1992) The Hilbert Space Method of Math and Physics Equations. Xi'an Jiaotong University Press, Xi'an. (In Chinese)

[8] Liu, B.Z. and Peng, J.H. (2004) Nolinear Dynamic. Higher Education Publishing House, Beijing. (In Chinese) 
Scientific Research Publishing (SCIRP) is one of the largest Open Access journal publishers. It is currently publishing more than 200 open access, online, peer-reviewed journals covering a wide range of academic disciplines. SCIRP serves the worldwide academic communities and contributes to the progress and application of science with its publication.

Other selected journals from SCIRP are listed as below. Submit your manuscript to us via either submit@scirp.org or Online Submission Portal.
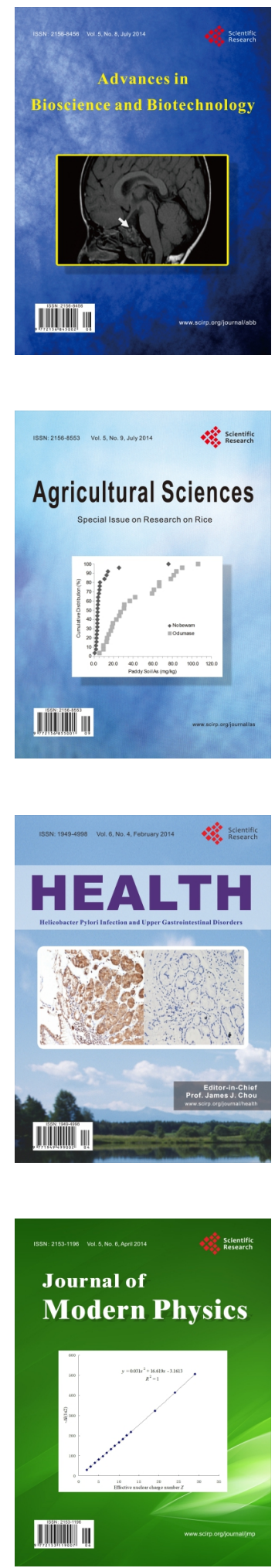
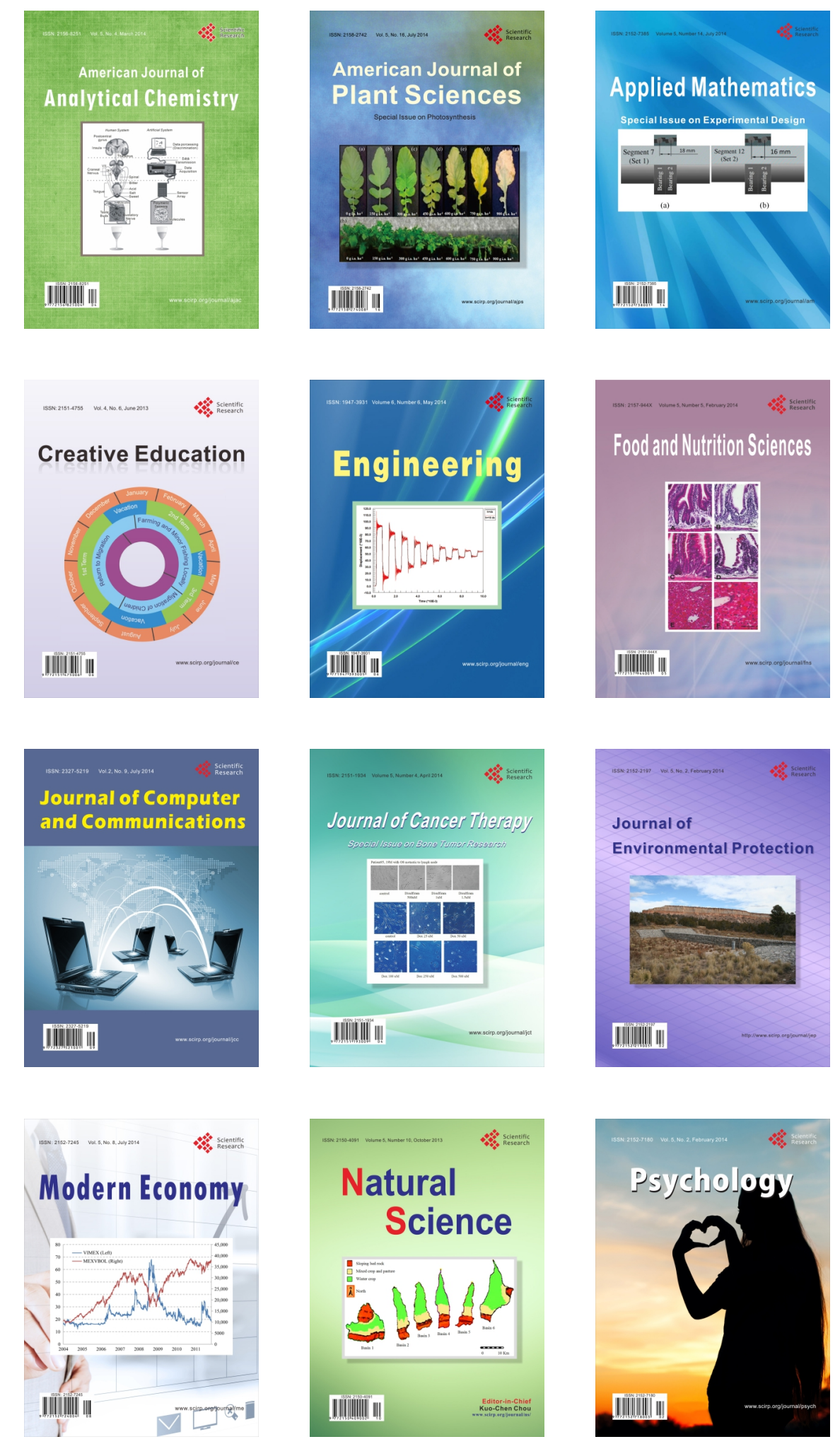